\title{
ANÁLISIS COMPARATIVO DE LA COMPETITIVIDAD DE LAS MIPYMES DE LOS SUBSECTORES COMUNICACIONES Y TRANSPORTES DEL ESTADO DE TABASCO'.
}

\author{
Juan José Chablé Sangeado*, Wendy Vannesa Oropeza Ruíz**.
}

\begin{abstract}
Chablé-Sangeado J.J., Oropeza-Ruíz W.V. Análisis comparativo de la competitividad de las MIPYMES de los subsectores comunicaciones y transportes del estado de Tabasco. Hitos de Ciencias Económico Administrativas 2012;18 (50):9-18.
\end{abstract}

\section{RESUMEN}

Objetivo: Analizar los principales factores competitivos que influyen en las micro, pequeñas y medianas empresas (MIPYMES) del sector comunicaciones y transportes del estado de Tabasco, para su crecimiento, expansión y permanencia en el mercado; así como su grado de competitividad con respecto al mismo sector en otros estados del país.

Material y método: El estudio fue dirigido a las MIPYMES de los subsectores de comunicaciones y transportes del estado de Tabasco y los resultados se obtuvieron de una muestra representativa aleatoria, 81 empresas, mismas que fueron analizadas por tamaño y permanencia. Como marco muestral se tomaron los censos económicos del INEGI 2004 y 2009, con los cuales se determinó la muestra de los subsectores, por medio de un muestreo aleatorio sistemático, y la recolección de datos se llevó a cabo a través de un cuestionario diseñado ex profeso que fue utilizado en las entrevistas. Para la comparación con otros estados del país, se tomaron los resultados de estudios semejantes desarrollados con la misma metodología que la presente investigación.

Resultados: Se comprueba que la competitividad de las MIPYMES de los subsectores de comunicaciones y transportes del estado de Tabasco, es semejante en comparación con los mismos subsectores de los estados tomados como referencia, dada la aceptación

\author{
Chablé-Sangeado J.J., Oropeza-Ruíz W.V. \\ Comparative analysis of the competitiveness of msmes \\ of the communications and transportation sectors in the \\ state of Tabasco. Hitos de Ciencias Económico \\ Administrativas 2012;18 (50):9-18.

\section{ABSTRACT}

Objective: Analyze the main competitive factors that influence the micro, small and medium enterprises (MSMEs) of the Communications and Transportation sector in the state of Tabasco, in relation to their growth, expansion and permanence in the market, as well as their competitiveness degree compared to the same sector in other states of the Mexican Republic.

Material and method: The study was carried out to MSMEs of the communications and transportation subsectors in the state of Tabasco and the results were obtained from a representative random sample, 81 companies that were analyzed by size and permanence. As a sampling frame, the INEGI 2004 and 2009 economic census were considered to determine the sample of the subsectors by a systematic random sampling methodology and the data collection was conducted through a questionnaire that was designed on purpose for the interviews. For comparisons with other states, results out of similar studies developed with the same methodology were taken.

Results: It was proved that the MSMEs competitiveness of the communications and transportation subsectors in the state of Tabasco is alike compared to the same subsectors in other states of the Mexican Republic that were taken as a reference, because of the acceptance

\footnotetext{
${ }^{1}$ Este trabajo forma parte de un proyecto de investigación sobre las MIPYMES de Tabasco.

* Doctor en Economía. Profesor-Investigador. División Académica de Ciencias Económico Administrativas. Universidad Juárez Autónoma de Tabasco (UJAT).

** Licenciada en Economía. División Académica de Ciencias Económico Administrativas. Universidad Juárez Autónoma de Tabasco (UJAT).
} 
de cuatro de las ocho hipótesis NULAS contrastadas en la investigación.

Conclusiones: El menor grado de escolaridad en Tabasco, de los directores/gerentes de los subsectores analizados constituye una desventaja competitiva. Sería conveniente mejorar los procesos de gestión de recursos humanos en el estado, ya que se encuentran a un nivel inferior que en las otras entidades del país; así mismo los estándares de calidad, y mejorar la capacidad de gestión financiera de las MIPYMES tabasqueñas de los subsectores en cuestión. of four out of the eight null hypotheses contrasted in the research.

Conclusions: The low education level of directors and managers of the analyzed subsectors in Tabasco is a competitive disadvantage. It would be desirable to improve the processes of human resource management in the state, as they are at a lower level than other states in the country, as well as the quality standards, and the financial management capacity of the MSMEs in the communication and transportation subsectors of Tabasco.
Palabras clave: Competitividad. Gestión financiera. Recursos humanos. Calidad e innovación.
Key words: Competitiveness. Financial management. Human resources. Quality and innovation. DIRECCIÓN PARA RECIBIR CORRESPONDENCIA: Correo electrónico: jjchable48@hotmail.com

a globalización de los mercados, la aceleración del cambio tecnológico y el desarrollo de nuevos sistemas de información y comunicación, unidas al escaso hábito de gestión del conocimiento y del capital humano, la dificultad para la captación de recursos externos, la menor calificación de empresarios y empleados, las dificultades de localización e infraestructura, y la complejidad para incorporar las innovaciones tecnológicas se consideran, entre otros, aspectos que dificultan y limitan el desarrollo de las micros, pequeñas y medianas empresas (Olivares, 2009).

Michael Porter (1991) dice que la prosperidad de una nación depende de su competitividad, la cual se basa en la productividad con la que ésta produce bienes y servicios. Políticas macroeconómicas e instituciones legales sólidas y políticas estables, son condiciones necesarias pero no suficientes para asegurar una economía próspera.

En México, el tipo de crecimiento económico seguido desde los años 40 hasta mediados de los 80 del siglo pasado, privilegió la industrialización vía la sustitución de importaciones y el crecimiento del mercado interno; mediante políticas proteccionistas y de subsidios, lo que permitió que se generara una planta industrial importante, formada principalmente por empresas manufactureras, las que llegaron a aportar, en su momento, más del $20 \%$ por ciento del PIB del país (Ruiz, C. y Zubirán C. 1992).
La micro, pequeña y mediana empresa en México representa más del $99 \%$ del total de las empresas establecidas y emplea al $72 \%$ de la fuerza laboral. No obstante, sufren graves deficiencias de organización, financiamiento y tecnología, que no les permiten abrirse a los mercados más competitivos.

De acuerdo con el Índice de Crecimiento de la Competitividad (ICC) del Word Economic Forum (WEF), México ha estado constantemente cayendo de posición competitiva, en el último reporte 20102011, México ocupa el lugar número 66 de 139 países analizados; es decir, el peor lugar en los últimos diez años, la mejor posición fue en el 2001, cuando se llego al lugar número 42.

En el caso específico de Tabasco según el Instituto Mexicano para la Competitividad A.C., se ubica en el lugar 29 del Índice de Competitividad Estatal 2010, misma posición que ocupaba en el índice 2008. Con su calificación actual (36/100), Tabasco aún se encuentra $17.2 \%$ debajo de la calificación promedio del país.

En este sentido, urge que las empresas tabasqueñas se actualicen y que el gobierno, los organismos de fomento se unan al reto de modernización y generación de más competitividad para conservar las fuentes de empleo existentes, crear más empleos y oportunidades inclusive con miras al mercado internacional. Además, se requiere de una decidida inversión en capital humano 
y en todos los niveles de la organización para poder sobrevivir en una economía totalmente globalizada.

Por lo anterior, se considera de sumo interés el conocer la situación de la competitividad de las MIPYMES de los diferentes sectores de la economía del estado de Tabasco, después de la catástrofe de 2007 y cómo se encuentra con respecto a otros estados referentes del país, en este caso especifico, el sector comunicaciones y transportes.

\section{MARCO TEÓRICO.}

Entre las teorías más reconocidas para el estudio de la competitividad se encuentra la de Michael E. Porter (1990) quién menciona que la competitividad de una empresa o grupo de éstas está determinada por seis dimensiones fundamentales: factores de oferta, de demanda, de integración a cadenas productivas, de estrategia empresarial, de oportunidades de mercado, y de apoyos institucionales; estos atributos y la interacción entre ellos explican por qué innovan y se mantienen competitivas las compañías ubicadas en determinadas regiones.

También Porter (1990) ha señalado dentro de sus estrategias competitivas que se debe desarrollar una amplia fórmula de cómo la empresa va a competir (ofensiva o defensivamente), cuáles deben ser sus objetivos y qué políticas son necesarias para alcanzar esos objetivos; para ello existen tres estrategias genéricas de éxito potencial para desempeñarse mejor que otras empresas:

1.El liderazgo total de costos se logra mediante un conjunto de políticas orientadas a este objetivo básico y se requiere de construir instalaciones capaces de producir grandes volúmenes en forma eficiente, de empeño en reducción de costos, de rígidos controles de costos y gastos indirectos, entre otros. 2.La diferenciación del producto o servicio es una estrategia que ofrece la empresa al crear algo que es percibido como único en el mercado. 3.El enfoque o alta segmentación consiste en enfocarse sobre un grupo de consumidores en particular, en un segmento de línea del producto o en un mercado geográfico.

Cabe mencionar la tipología de estrategia competitiva (Miles y Snow, 1978) que se utiliza como criterio para clasificar en las empresas la actitud hacia el grado de innovación (de producto, servicio o mercado) que presentan. Esta clasificación distingue cuatro tipos de empresas:

1) Las exploradoras, son aquellas que realizan cambios y mejoras en los productos y mercados con relativa frecuencia, tratando de ser las primeras en desarrollar nuevos productos, aun con el riesgo de que estas innovaciones no tengan éxito. 2) Las analizadoras, mantienen una base relativamente estable de productos y mercados, mientras que desarrollan de forma selectiva nuevos productos y mercados, tratando de imitar a las empresas que ya los desarrollaron y tuvieron éxito. 3) Las defensivas, que ofrecen un conjunto relativamente estable de productos para un mercado relativamente estable. No se interesan en las modificaciones sino que se concentran en la mejora continua del trabajo dentro de su campo de actuación. 4) Sin estrategia definida, son empresas que no cuentan con un área de producto-mercado duradera y estable. Normalmente actúan forzadas por las presiones del entorno y de la competencia.

Diversos estudios señalan algunos factores explicativos del éxito competitivo dentro de los que podemos mencionar la planeación estratégica que se considera un aspecto clave para el éxito y rentabilidad de la empresa (Chandler, 1962 y Ansoff, 1965); las alianzas de cooperación en las empresas representan una alternativa estratégica que permite alcanzar el tamaño competitivo en ciertas actividades, sin necesidad de fusionarse y por tanto manteniendo la independencia jurídica y patrimonial (Aragón y Rubio, 2005a); estructura organizativa flexible es la capacidad de respuesta de la empresa para adaptarse o para anticiparse a los continuos cambios que afectan a las organizaciones. La flexibilidad organizacional está muy relacionada con la innovación y con el éxito competitivo (Díez de Castro et al., 2002); los recursos humanos es de los principales factores que se destacan como explicativos de la competitividad empresarial. Los conocimientos, habilidades y actitudes de los trabajadores y cómo estos trabajan son aspectos cada vez más importantes para las empresas (Pfeffer, 1994).

Se consideran relevantes por diversos autores: la innovación y la tecnología como uno de los pilares básicos de la competitividad de la empresa (Bueno y Morcillo, 1994). Con respecto a la calidad se coincide en señalar que las habilidades relacionadas a ésta son 
fundamentales para la competitividad y el éxito de las empresas (Viedma, 1990; Luck, 1996). Además, se recomienda fomentar el uso de las nuevas tecnologías de la información y comunicación para la gestión de la empresa (Viedma, 1990; Llopis, 2000; Donrrosoro et al., 2001) y se considera que los recursos financieros son clave para aumentar las posibilidades de acometer las inversiones necesarias para el desarrollo de la organización y crear una base financiera más sólida, (Aragón y Rubio, 2005b). También se hace preciso tener un alto conocimiento de la oferta financiera de las diferentes fuentes y saber aprovechar los programas de ayudas oficiales existentes (Chable, J., et al. 2010).

\section{MATERIAL Y MÉTODO.}

El tipo de investigación que se realizó fue: aplicada, documental y de campo In situ, no experimental o expost-facto y descriptivo y correlacional. La población objeto de estudio está conformada por micro, pequeñas y medianas empresas de los subsectores comunicaciones y transportes del estado de Tabasco.

El criterio de selección fue mediante un muestreo aleatorio sistemático, clasificando a las empresas por tamaños según el número de empleos generados: microempresas (más de 4 y menos de 11 trabajadores), pequeñas empresas (de 11 a 50 trabajadores) y empresas medianas (de 51 a 250 trabajadores); y como marco muestral se tomó el censo económico levantado por el INEGI con datos de 2004, actualizado posteriormente con el censo económico 2009 cuando estuvo disponible.

Los estudios que se tomaron como punto de comparación fueron los realizados por la Universidad Autónoma de Aguascalientes (2007), la Universidad Autónoma de Durango (2007), la Universidad Autónoma de Nayarit (2007) y la Universidad Autónoma de Veracruz (2007), realizados con la misma metodología que el presente.

El sector empresarial en Tabasco suma, según datos del censo económico de 2009, un total de 52 mil 663 negocios, en donde las microempresas (hasta 10 empleados) constituyen el $94.33 \%$, le siguen en importancia las pequeñas (de 11 a 50 ) con el $4.55 \%$ de la representación total, menos del $1 \%$ de medianas (de 51 a 250) y el resto de grandes.
No obstante que las MIPYMES en Tabasco constituyen el $99.82 \%$ de la planta productiva empresarial, adolecen, como pasa a nivel nacional, de una serie de limitaciones y problemas como: atraso tecnológico, teniendo maquinarias de segunda o tercera mano, desventajas en el acceso a los mercados de insumos y productos finales, bajo uso de técnicas modernas de administración: usando muchas veces ideas para diseñar su empresa de lo que ve y copiando muchas veces modelos obsoletos, niveles deficientes de calidad, dificultades en el acceso a crédito institucional y a la información económica relevante.

La fórmula para determinar el tamaño de la muestra que se utilizó es la siguiente:

Diseño estadístico:

$$
n_{i}=\frac{4 p q N}{\left[e^{2}(N-1)+4 p q\right]}
$$

En donde:

$\mathbf{n}_{\mathrm{i}}=$ tamaño de la muestra a estimar de los subsectores;

py q: son las proporciones de respuestas dicotómicas clave que para mejor representatividad se considera 0.5 ;

$\mathbf{N}=$ población del universo de los subsectores de empresas censadas que resultó de 428;

$\mathbf{e}=$ error de precisión adoptado, para cada caso fue el máximo del $10 \%$; cálculo que arrojó un total de 81 encuestas a levantar (suma de las $n_{i}$ ) como se muestra a continuación:

$$
\begin{gathered}
n_{i}=\frac{4(0.5)(0.5) 428}{\left[(0.10)^{2}(428-1)+4(0.5)(0.5)\right]}= \\
\frac{4(428)}{\left[(0.01)^{2}(427)+1\right]}=\frac{428}{[5.27]}=81.21
\end{gathered}
$$

El tamaño de la muestra determinado es de 81 empresas. La recopilación de información fue mediante entrevistas a los directores o gerentes de las MIPYMES seleccionadas. 
El objetivo general consistió en analizar cuáles son los principales factores competitivos que influyen en las MIPYMES de los subsectores comunicaciones y transportes en el estado de Tabasco, para su crecimiento, expansión y permanencia en el mercado; así como, comparar su competitividad con respecto a otros estados del país.

Los objetivos específicos fueron: Conocer las principales características generales de las MIPYMES de los subsectores comunicaciones y transportes, analizar las estrategias y factores de la competitividad de los subsectores comunicaciones y transportes, conocer su estructura organizativa y la gestión de recursos humanos, indagar como están sus indicadores de tecnología, calidad e innovación, conocer cuál es el uso que se le está dando actualmente a las Tecnologías de Información y Comunicación TIC, analizar sus prácticas contables y financieras en comparación con otros estados del país.

Las hipótesis para las contrastaciones estadísticas de la investigación que se plantearon fueron: hipótesis nula que considera que la competitividad de las MIPYMES de los subsectores comunicaciones y transportes de la economía del estado de Tabasco es similar a la de otros estados del país tomados como referencia, porque los factores que influyen en ella son equivalentes.

Las hipótesis secundarías se hicieron con respecto a las siguientes ocho variables: El nivel de estudios del director o gerente, la planeación estratégica, los procesos de gestión de recursos humanos, la posición tecnológica, los estándares de calidad, la capacidad de innovación, el uso de tecnología moderna de información y comunicación y la capacidad de gestión financiera.

Considerando a las MIPYMES de los subsectores comunicaciones y transportes en el estado de Tabasco en un nivel bajo en comparación con otros estados del país tomados como referencia.

En la obtención de los tamaños de las muestras de los estados que se toman como referencia en esta investigación, el procedimiento que se siguió fue tomar los resultados de estudios análogos sobre las micro, pequeñas y medianas empresas de los subsectores comunicaciones y transportes del sector terciario de los estados donde ya se han realizado investigaciones con la misma metodología, como son: Aguascalientes, Nayarit, Durango y Veracruz.

El estadístico de prueba que se utilizó para contrastar las hipótesis de trabajo fue la diferencia de proporciones para cada variable especificada en las hipótesis secundarias, al comparar a Tabasco con otros estados del país tomados como referencia (Aguascalientes, Durango, Nayarit y Veracruz). A excepción de las hipótesis seis y ocho, las cuales se trabajaron con medias y como no se contó con las varianzas de las muestras de los otros estados, se optó por hacer contrastaciones de tipo cualitativo para aceptar o rechazar las hipótesis nulas planteadas.

Cabe mencionar que este estudio, trata de probar, que cada uno de los factores explicativos del éxito competitivo en el estado de Tabasco son bajos, débiles o deficientes con respecto a los estados de referencia (Aguascalientes, Durango, Nayarit y Veracruz). Por lo tanto, así se plantean las hipótesis estadísticas alternativas y se les graficó sobre la distribución Z, con una sola región de rechazo en la zona izquierda (Figura 1).

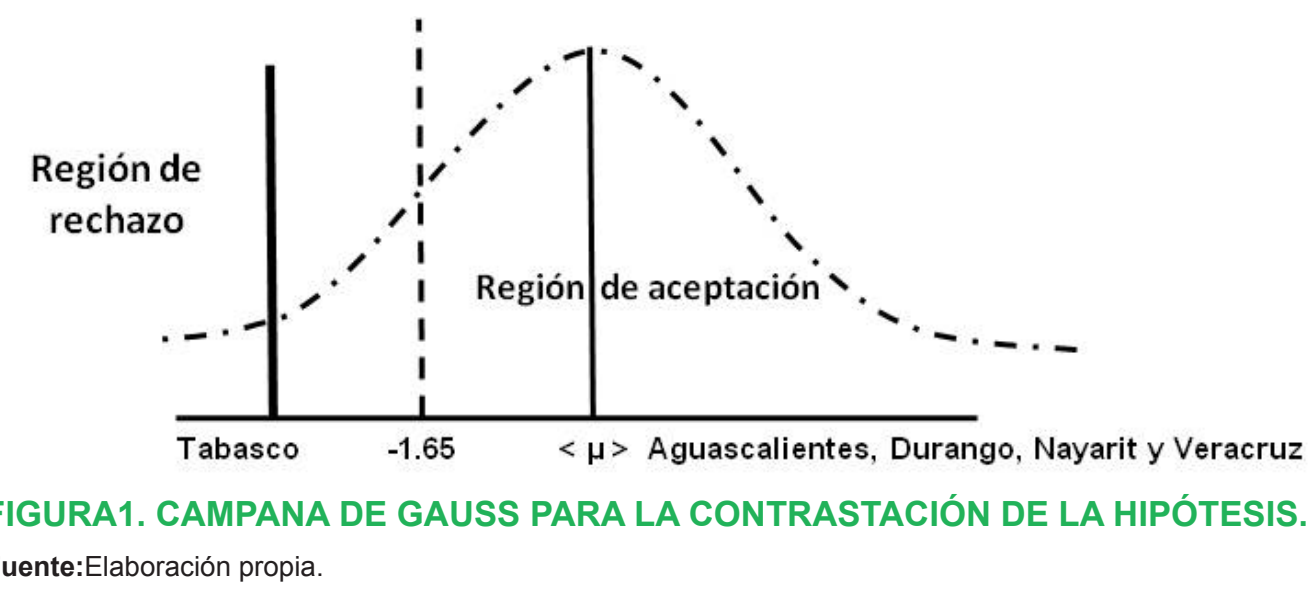


TABLA I. MUESTRA DE LOS ESTADOS DE REFERENCIA.

\begin{tabular}{ccccccc}
\hline Estados & $\begin{array}{c}\text { Tabasco } \\
\text { (Sur) }\end{array}$ & $\begin{array}{c}\text { Durango } \\
\text { (Norte) }\end{array}$ & $\begin{array}{c}\text { Nayarit } \\
\text { (Oeste) }\end{array}$ & $\begin{array}{c}\text { Aguascalientes } \\
\text { (Centro) }\end{array}$ & $\begin{array}{c}\text { Veracruz } \\
\text { (Este) }\end{array}$ & Total \\
\hline $\begin{array}{c}\text { No. de } \\
\text { empresas }\end{array}$ & 81 & 25 & 11 & 8 & 36 & $\mathbf{8 0}$ \\
(frecuencia) & & & & & & \\
\hline
\end{tabular}

Nota: Elaboración propia con datos obtenidos de la base del proyecto de investigación, «Análisis comparativo de la competitividad del sector empresarial tabasqueño por ramas de actividad económica» FOMIXCONACYT - Gobierno del Estado. Clave TAB-2009-C17-120751. 2010 e informes finales de investigación de los estados de Aguascalientes, Nayarit, Durango y Veracruz.

En consecuencia se necesitaba para la prueba de hipótesis entre dos proporciones una $n_{p}$ lo cual se obtuvo como sigue:

Se sumaron todas las muestras (MIPYMES de los subsectores comunicaciones y transportes) de los estados: Durango, Nayarit, Aguascalientes y Veracruz, y se obtiene $n_{p}=80$ (Tabla I).

\section{ANÁLISIS Y DISCUSIÓN.}

Las características de las organizaciones que conforman el estudio señalan en cuanto al tamaño que $40.7 \%$ lo constituyen las microempresas, $43.2 \%$ las pequeñas empresas y $16.1 \%$ las medianas empresas. El $24.7 \%$ son empresas que tienen 10 años o menos establecidas en el mercado, mientras el resto $(75.3 \%)$ tienen más de 10 años de fundación. El mercado al cual ofertan su servicio es $64.1 \%$ local, $26.3 \%$ estatal, 7.6 nacional y sólo el $2 \%$ restante es internacional.

Las características de los individuos que dirigen las empresas y que mayormente proporcionaron la información son las siguientes: gerentes jóvenes (menor e igual que 40 años) el $27.2 \%, 42 \%$ son personas entre 41 y 55 años y $30.9 \%$ mayor a 50 años; su nivel de estudios es en su mayoría licenciatura (43.2\%), seguido por estudios básicos con 27.1, posteriormente con bachillerato $19.8 \%$ y por último, técnico superior universitario con $9.9 \%$.

Los resultados descriptivos encontrados en las empresas de la muestra estudiada presentan datos interesantes.
En cuanto a la estrategia competitiva de Miles y Snow (1978), de los cuatro tipos de actividades reportadas predominan las analizadoras, ya que las empresas tratan de mantener la línea de servicio que prestan y proponer nuevas pero imitando a otras que les haya funcionado. La estrategia menos adoptada es la reactiva; es decir, sólo un porcentaje pequeño actúa forzada por el entorno y sin estrategia.

Respecto a la tipología de Porter (1999), la estrategia más utilizada son los costos inferiores a la competencia, notando el intento por reducir los gastos, y que es utilizada en mayor medida por micro y medianas empresas. La menos practicada es la diferenciación del servicio; es decir, muy pocas empresas buscan ofrecer un servicio o producto que se considere único en el mercado.

Los datos sobre la realización de planeación estratégica nos reflejan que sólo $47 \%$ la realiza y más del $90 \%$ a corto plazo ya que la incertidumbre en el entorno económico es muy alta.En el mismo sentido la mayor parte de las empresas no realizan alianzas o acuerdos de ninguna índole con otras empresas $(75.6 \%)$ y las pocas que la practican se inclinan por hacerlo en temas de logística.

En lo que concierne a la flexibilidad organizacional se externalizan trabajos a otras empresas en mayor porcentaje que contratar empleados temporales dentro de la empresa. En las actividades relacionadas con los recursos humanos, las más realizadas son la capacitación del personal y el reclutamiento y selección de personal. 
Además, existe una gran necesidad de departamentos en las MIPYMES del estado de Tabasco, sobre todo los concernientes a investigación y desarrollo, calidad y recursos humanos. Este último reflejado en la gran necesidad de aplicación de actividades de administración de recursos humanos

Por lo que corresponde a la posición tecnológica el mayor porcentaje $(32.1 \%)$ reconoce estar en una posición sostenible; es decir, utilizan tecnología idéntica a la de las otras empresas y sólo realizan nuevas inversiones si han funcionado en éstas. Lo cual se refleja en el hecho de que la realización de compras y ventas por internet sea muy poca, ya que son los menores porcentajes dentro de la implantación de las tecnologías de la información y comunicación.

Dentro de las innovaciones se observa que es de suma importancia para las empresas que la realizan, aunque las que realizan más del $50 \%$ de las empresas son en el proceso del servicio, adquisición de nuevos bienes de equipo y mejoras en servicios existentes. Dejando atrás las relacionadas con la dirección y gestión y comercializar nuevos productos.

\section{RESULTADOS.}

La primera hipótesis examina el nivel de estudios de los gerentes de las MIPYMES del estado versus estados referentes (Aguascalientes, Durango, Nayarit y Veracruz). La proporción del estado de Tabasco $\left(Z_{c}=\right.$ $-1.70)$ contra los estados referentes $\left(Z_{t}=-1.65\right)$ muestra una diferencia significativa y una inclinación hacia el área de rechazo, lo que indica que se acepta que el nivel educativo de los gerentes de las MIPYMES de los subsectores comunicaciones y transportes en el estado de Tabasco, es bajo en comparación con otros estados del país, a un nivel de confianza del 95\%.

La segunda hipótesis, valida la relación entre las prácticas de planeación de las empresas del estado y las de los otros estados ya mencionados, lo cual muestra una $Z_{c=}-0.709$ que cae dentro del área de aceptación de la hipótesis nula; entonces se puede decir que las prácticas de planeación estratégica de las MIPYMES de los subsectores comunicaciones y transportes de Tabasco es semejante a las de los otros estados referenciados, a un nivel de confianza del $95 \%$.
La tercera hipótesis nula, se refiere a la aplicación de los procesos de recursos humanos en las empresas y la diferencia entre la proporción estatal contra la de los otros estados del país $\left(Z_{t}=-1.65\right)$ es significativa y se inclina hacia el área de rechazo para el reclutamiento y selección del personal $\left(Z_{c}=-1.93\right)$, la promoción del personal $\left(Z_{\mathrm{c}}=-2.33\right)$ y los programas de incentivos $\left(Z_{c}=-1.83\right)$. El caso contrario se acepta para la capacitación del personal $\left(Z_{c}=-0.32\right)$ y la evaluación del desempeño $\left(Z_{c}=-1.51\right)$; por lo tanto, los procesos de gestión de recursos humanos de las MIPYMES de los subsectores comunicaciones y transportes del estado de Tabasco, son menos eficientes en comparación con los estados mencionados anteriormente.

La cuarta hipótesis, explora la posición tecnológica de las MIPYMES del estado contra los otros estados y la diferencia entre la proporción estatal $\left(Z_{c}=-0.084\right)$ contra los estados referentes $\left(Z_{t}=-1.65\right)$ no es estadísticamente significativa y se inclina hacia el área de aceptación; por lo que se puede concluir que la posición tecnológica de los subsectores transporte y comunicaciones de Tabasco es similar al de otros estados del país, a un nivel de confianza del $95 \%$.

La quinta hipótesis, se refiere a los estándares de calidad de las MIPYMES estudiadas y puesto que existe diferencia significativa entre las proporciones de las poblaciones estudiadas se rechaza la hipótesis nula, ya que $Z_{c}=-5.92$ cae fuera del área de aceptación de la hipótesis nula $\left(Z_{t}=-1.65\right)$, entonces se puede decir, que en cuanto a los estándares de calidad, las MIPYMES de los subsectores transporte y comunicaciones de Tabasco son deficientes.

La sexta hipótesis evalúa la capacidad de innovación de las MIPYMES en el estado se encontró poca diferencia significativa existente entre las medias de Tabasco y de los estados estudiados en la realización de innovaciones, en una escala de uno a cinco; por lo anterior, se considera que la capacidad de innovación de las MIPYMES de los subsectores estudiados del estado de Tabasco es equivalente en comparación con otros estados tomados como referencia.

La séptima hipótesis examina el uso de las tecnologías modernas de información y comunicación de las MIPYMES de comunicaciones y transportes en el estado. Con una diferencia significativa se rechaza la hipótesis nula sobre el uso de tecnologías modernas 
con respecto al correo electrónico $\left(Z_{c}=-3.09\right)$, pero se aceptan aquellas referentes a la página web $\left(Z_{c}=-1.20\right)$ y a la mercadotecnia por medio de Internet $\left(Z_{c}=-0.049\right)$, con un nivel de confianza del 95 por ciento. Por lo cual se concluye que el uso de tecnologías modernas de información y comunicación de las MIPYMES de los subsectores comunicaciones y transportes del estado de Tabasco es equivalente al de los otros estados.

Finalmente, está la prueba que se refiere a la capacidad de gestión financiera de las MIPYMES de los subsectores de comunicaciones y transportes en el estado. Los hallazgos encontrados revelan que las diferencias, entre las medias de Tabasco y de los estados referentes que ocupan los primero lugares en el uso de financiamiento bancario en activos fijos y circulantes, son estadísticamente significativas; por lo anterior, la gestión financiera de las MIPYMES de estos subsectores en el estado de Tabasco es deficiente en comparación con los otros estados; por lo tanto, se acepta la octava hipótesis secundaria.

\section{CONCLUSIONES.}

Con respecto a las seis hipótesis nulas analizadas estadísticamente, tres fueron aceptadas: las referentes a la planeación estratégica, a la posición tecnológica ya las tecnologías de la información y comunicación, lo que indica situaciones de equivalencia de la competitividad empresarial tabasqueña y las tres restantes se rechazaron: sobre el nivel educativo del gerente, los procesos de recursos humanos y la certificación de calidad, lo que hace pensar en desventajas de las MIPYMES tabasqueñas en estos aspectos.

Con respecto a las hipótesis secundarias analizadas cualitativamente, se aceptó una que es sobre la capacidad de gestión financiera; es decir, se está en desventaja y se rechazó la hipótesis secundaria de la capacidad de innovación, esto es que si se está a un nivel semejante de competitividad en este aspecto con otros estados del país.

En consecuencia, no hay una conclusión definitiva sobre la competitividad de estos subsectores de la economía tabasqueña, pero es semejante en comparación con otros estados del país, ya que en el mismo número de factores competitivos importantes, las MIPYMES locales aparentemente están igual que en los estados tomados como referencia, y en otros tantos están en desventaja.

Por otro lado, se sugieren acciones convenientes derivadas del estudio:

1.-Cursos de capacitación para elevar el nivel de conocimientos gerenciales de los empresarios y los operarios de los subsectores analizados.

2.-Mejorar los procesos de gestión de recursos humanos donde resultaron menos competitivas las MIPYMES tabasqueñas.

3.- Elevar los estándares de calidad del servicio que prestan estas empresas.

4.-Mejorar la gestión financiera y los flujos de información entre las MYPIMES estudiadas y las fuentes de financiamiento, tanto públicas como privadas.

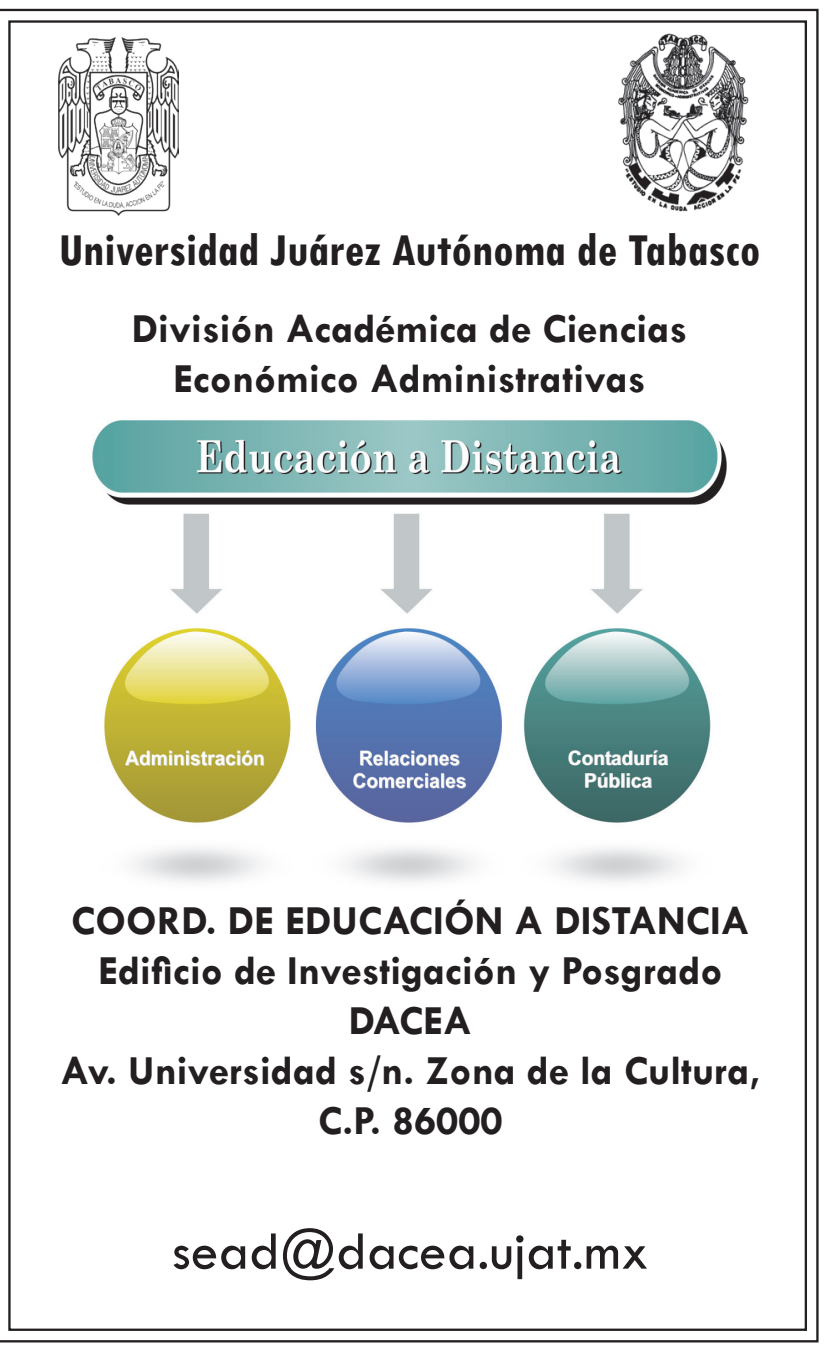

HITOS DE CIENCIAS ECONÓMICO ADMINISTRATIVAS 


\section{REFERENCIAS}

Aragón, A. y Rubio, A. (2005a). Factores asociados con el éxito competitivo de las MIPYME industriales en España. Universia Bussiness Review, (8), 38-51.

Aragón, A. y Rubio, A. (mayo-agosto 2005b). Factores explicativos del éxito competitivo: el caso de las MIPYMES del Estado de Veracruz. Contaduría y Administración, (216), 35-69.

Chablé, J.J. (2006). La importancia de la Información Financiera en el acceso al Crédito de Micro y Pequeñas Empresas Manufactureras en Tabasco. (Tesis Doctoral) México D.F. UNAM.

Chablé, J.J. y Aragón, A. (2009). Análisis estratégico para el desarrollo de la micro, pequeña y mediana empresa de Tabasco. Universidad Juárez Autónoma de Tabasco. Villahermosa, Tabasco.

Chablé, J.J. et al. (2010). El mercado del crédito de MYPES en el estado de Tabasco. Universidad Juárez Autónoma de Tabasco. Villahermosa, Tabasco.

Díez de Castro, J., Redondo, C., Barreiro, B., y López, M.A. (2002). Administración de empresas. Dirigir en la sociedad de la información. Madrid, España: Pirámide.

Informe MPYME Iberoamérica. (2009). Análisis estratégico para el desarrollo de la MPYME en lberoamérica (FAEDPYME). Editores: Universidad Politécnica de Cartagena y FAEDPYME.

Instituto Mexicano para la Competitividad (IMCO). Tabasco, análisis de la competitividad 2010. Documento elaborado por él. Recuperado de, http://imco.org.mx/indice_estatal_2010/ PDFS/27.Tabasco.pdf 24-10-10

Instituto Nacional de Estadística, Geografía e Informática. (2009). Censo Económico 2009. México.

Instituto Nacional de Estadística, Geografía e Informática. (2001). Micro Pequeña, Mediana y Gran Empresa. Estratificación de los establecimientos, censos económicos 1999. Aguascalientes, México.

Llopis, J. (2000). Dirigiendo: 11 factores claves del éxito empresarial. Barcelona, España: Ediciones Gestión 2000.

Lombana, J. y Rozas, S. (2009). Marco Analítico de la Competitividad: Fundamentos de Estudio de la Competitividad Regional. Pensamiento y Gestión. Universidad del Norte, (26), 1-38.

Miles, R.E. y Snow, C.C. (1978). Organizational strategy structure and process. New York, U.S.A: McGraw-Hill.

Olivares, O. (2009). Competitividad y calidad factores críticos que contribuyen al éxito de Empresas de Menor Tamaño en mercados globales de país emergente. Revista Chilena de Economía y Sociedad, 3, (1), 34-35.

Porter, MichaelE. (1990). Ventaja competitiva. México: Porrúa.

Porter, M. E. (1999). Estrategia competitiva. Técnicas para el análisis de los sectores industriales y de la competencia. México: Compañía Editorial Continental (CECSA).

Rojas, R. (2000). Guía para realizar investigaciones sociales. México. Plaza y Valdez.

Ruiz, C.y Dussel, E. (1999). Dinámica regionaly competitividad industrial. México D.F.: Jus S.A. de C.V.

Ruiz, C. (Coordinador) (2002). Desarrollo empresarial en América Latina. México D.F.: Nacional Financiera-UNAM.

Ruiz, C. (1996). Economía de la Pequeña Empresa. México D.F.: Ariel.

Salvatore, D. (1991). Econometría. México, D.F.: Mc Graw-Hill.

Secretaría de Comunicaciones y Transportes. (2009). Anuario estadístico del Sector Comunicaciones y Transportes. México.

Secretaría de Comercio y Fomento Industrial. (1999). Clasificación de las micro y pequeñas empresas. Diario Oficial de la Federación. 30 de marzo, México, D.F.

Universidad Autónoma de Aguascalientes. (2007). Análisis estratégico para el desarrollo de la Micro, Pequeña y Mediana Empresa del estado de Aguascalientes. Aguascalientes, México. Autor.

Universidad Autónoma de Durango. (2007). Análisis estratégico para el desarrollo de la Micro, Pequeña y Mediana Empresa del estado de Durango. Durango, México. Autor.

Universidad Autónoma de Nayarit. (2007). Análisis estratégico para el desarrollo de la Micro, Pequeña y Mediana Empresa del estado de Nayarit. Nayarit, México. Autor.

Universidad Autónoma de Veracruz. (2007). Análisis estratégico para el desarrollo de la Micro, Pequeña y Mediana Empresa del estado de Veracruz. Xalapa, Veracruz, México. Autor.

World Economic Forum (WEF) (2010-2011). Reporte del Índice de Crecimiento de la Competitividad (ICC). Recuperado en: http://www.weforum.org/gcr 24-10-10. 
Notivjat

Desde Ia Música

CL Target

= 5 olo 10

: Agroujat

Transporte Público

Relax

EI Guentero

Entre Niños Ujat

Territorio Juchimán

En boncierto Ujat

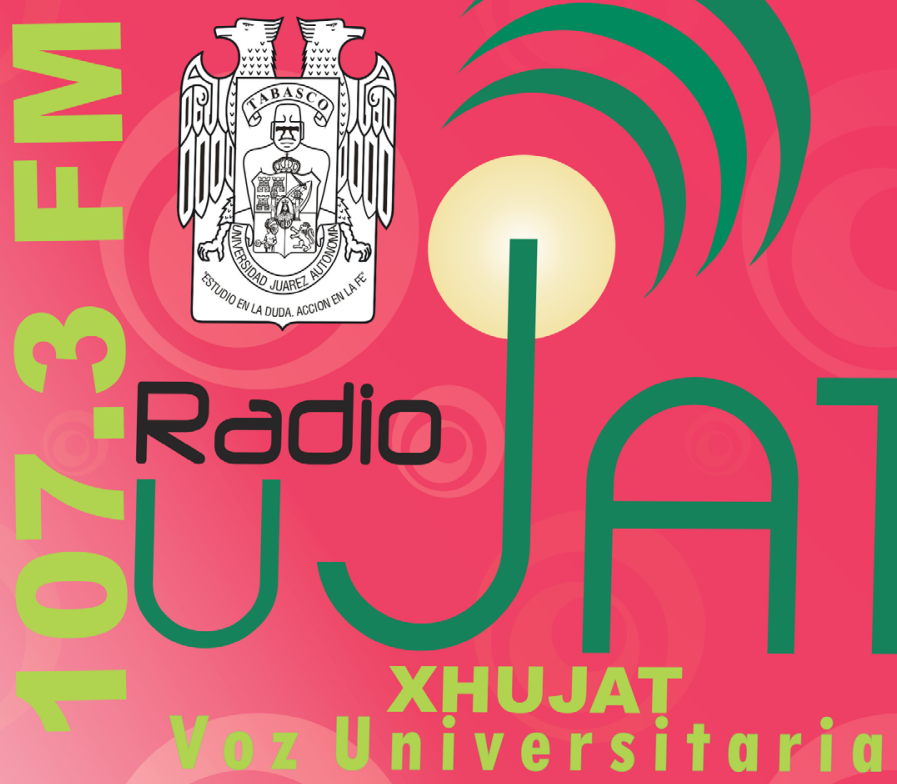

(B) 3141141

W www.radio.vjat.mx

f radio ujat

B @RADIO_UJAT

Dradioujatenvivo@hotmail.com

Música Noticias Deportes Cultura Ciencia -Tecnología

"Por la universidad decalidad"

\section{HITOS DE CIENCIAS ECONOMICO ADMINISTRATIVAS}

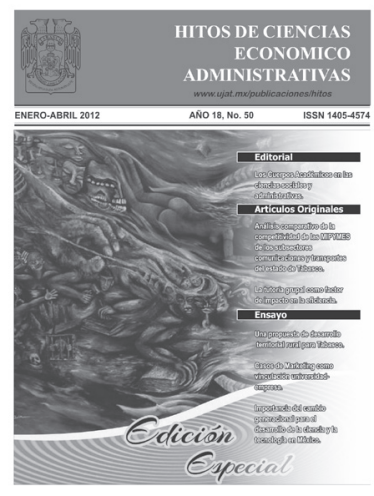

www.ujat.mx/publicaciones/hitos
Revista que publica cuatrimestralmente la DACEA-UJAT, se encuentra dentro del LATINDEX (Catálogo de Revistas Latinoamericanas) desde el mes de diciembre del año 2000, e indizada en CLASE (base de datos bibliográfica en Ciencias Sociales y Humanidades de la Universidad Nacional Autónoma de México) a partir del 23 de enero del 2001.

\section{http://www.dgbiblio.unam.mx}

Actualmente, nuestra publicación periódica está totalmente digitalizada y la puedes encontrar en la página WEB de la UJAT y es posible accesar a ella a través de: 